\title{
Five-Step Synthesis of Yaequinolones J1 and J2
}

Johannes Schwan, Merlin Kleoff, Philipp Heretsch* and Mathias Christmann*

Freie Universität Berlin, Institut für Chemie und Biochemie, Takustraße 3, 14195 Berlin, Germany

\section{Supporting Information}

General Methods
Synthesis of Compounds
${ }^{1} \mathrm{H}$ and ${ }^{13} \mathrm{C}$ NMR comparisons
References




\section{General Methods}

Analytical data were obtained using the following equipment:

NMR spectroscopy: ${ }^{1} \mathrm{H}$ and ${ }^{13} \mathrm{C}$ NMR spectra were acquired on a JEOL ECP 500 (500 MHz), BRUKER Avance 500 (500 MHz), VARIAN Inova 600 (600 MHz), and a BRUKER Avance 700 (700 MHZ) in the reported deuterated solvents. The chemical shifts were reported relative to the deuterated solvents' residual shifts. The multiplicities of the signals are reported using the following abbreviations: $s=$ singlet, $\mathrm{d}=$ doublet, $\mathrm{t}=$ triplet, $\mathrm{q}=$ quartet, $\mathrm{p}=$ quintet, $\mathrm{br}=$ broad and combinations thereof.

The spectra were processed with the software MESTREC 9.0.

Mass spectra were obtained on a ESI-FTICR-MS: Ionspec QFT-7 (Agilent/Varian)

IR spectroscopy: IR Spectra were recorded on a JASCO FT/IR-4100 spectrometer. Characteristic absorption bands are reported in wavelengths $\tilde{\mathrm{V}}$ in $\mathrm{cm}^{-1}$ and were analyzed with the software Spectral Manager from JASCO.

Flash column and thin layer chromatography: Reaction progress was monitored by thin layer chromatography (silica gel $60 \mathrm{~F} \mathrm{254}$, E. Merck) using UV light $(\lambda=254 \mathrm{~nm}$ ) for visualization or vanillin staining agent ( $170 \mathrm{~mL}$ methanol, $20.0 \mathrm{ml}$ conc. acetic acid, $10.0 \mathrm{~mL}$ conc. sulfuric acid, $1.0 \mathrm{~g}$ vanillin). Flash column chromatography was performed using silica gel M60 from MACHEREY \& NAGEL (particle size: $40-63 \mu \mathrm{m})$.

HPLC was conducted on a modular KNAUER HPLC system with a UV detector at $254 \mathrm{~nm}$ and differential refractometer. For chiral separations, a ChiralPAK® IA (Daicel) $32 \times 250 \mathrm{~mm}$ column was used.

MPLC was performed with a TELEDYNE ISCO Combi-Flash Rf or a TELEDYNE ISCO Combi-Flash $\mathrm{Rf} 200$ using prepacked $\mathrm{SiO}_{2}$-columns and cartridges from TELEDYNE. UV response was monitored at $254 \mathrm{~nm}$ and $280 \mathrm{~nm}$. As eluents, cyclohexane (99.5\%+ quality) and EtOAc (HPLC grade) were used.

Optical rotations were measured on a JASCO P-2000 polarimeter at $589 \mathrm{~nm}$ using $100 \mathrm{~mm}$ cells and the solvent and concentration $(\mathrm{g} / 100 \mathrm{~mL})$ indicated

Microwave reactions: A BIOTAGE Initiator+ microwave reactor (400 W) was used to perform microwave heated reactions. Reactions were conducted in $5 \mathrm{~mL}$ glas reactors from BIOTAGE. The temperature was monitored using the IR thermometer of the microwave and power was adjusted automatically to maintain the temperature constant.

Reagents and solvents: Reactions with air or moisture sensitive substances were carried out under an argon atmosphere using standard Schlenk techniques. All reagents and solvents were used as purchased from commercial suppliers unless otherwise noted. Anhydrous solvents were purified with the solvent purification system MB-SPS-800 (BRAUN). Dry acetonitrile was purchased from ACROS Organics in AcroSeal ${ }^{\odot-b o t t l e s ~ u n d e r ~ a r g o n ~ a t m o s p h e r e ~ w i t h ~ m o l e c u l a r ~ s i e v e s ~(~} 3 \AA$ ). HPLC-grade acetonitrile was purchased from Fischer Scientific. The solvents used for column chromatography (EtOAc, $n$-pentane, $n$-hexane) and work up were purified from commercially available technical grade 
solvents by distillation under reduced pressure with the help of rotatory evaporators (HEIDOLPH or IKA) at $40^{\circ} \mathrm{C}$ bath temperature.

2-Bromoresorcinol was purchased from SIGMA ALDRICH. Imide 4 was prepared according to ref. 1. Compound names are derived from CHEMDRAW 18.0 and are not necessarily identical with the IUPAC nomenclature.

Room temperature refers to $23^{\circ} \mathrm{C}$. 


\section{Synthesis of Compounds}

\section{Optimization of the Tandem Knoevenagel-Electrocyclization ${ }^{a}$}

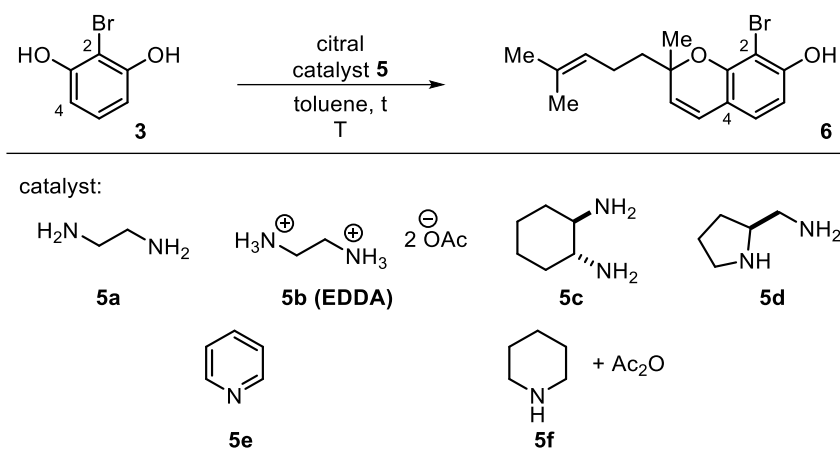

\begin{tabular}{llllll}
\hline \hline Entry & $\begin{array}{l}\text { Catalyst 5 } \\
{[\mathrm{mol} \%]}\end{array}$ & $\begin{array}{l}\text { Time } \\
{[\mathrm{h}]}\end{array}$ & $\begin{array}{l}\text { Temp. } \\
{\left[{ }^{\circ} \mathrm{C}\right]}\end{array}$ & $\begin{array}{l}\text { Citral } \\
\text { [equiv.] }\end{array}$ & $\begin{array}{l}\text { Yield } \\
{[\%]}\end{array}$ \\
\hline 1 & a [30] & 0.5 & 150 & 1.5 & 26 \\
2 & $\mathbf{b}[30]$ & 0.5 & 150 & 1.5 & 33 \\
3 & $\mathbf{c}[30]$ & 0.5 & 150 & 1.5 & 8 \\
4 & $\mathbf{d}[30]$ & 0.5 & 150 & 1.5 & 7 \\
$5 \mathrm{~b}$ & $\mathbf{e ~ [ 2 0 0 ]}$ & 18 & 90 & 1.0 & 0 \\
$6^{\mathrm{b}, \mathrm{c}}$ & $\mathbf{f}[220]$ & 40 & 130 & 1.0 & 11 \\
7 & $\mathbf{b}[10]$ & 5 & 140 & 1.0 & 29 \\
8 & $\mathbf{b}[10]$ & 5 & 140 & 1.5 & 37 \\
9 & $\mathbf{b}[10]$ & 5 & 140 & 2.0 & 42 \\
10 & $\mathbf{b}[10]$ & 5 & 140 & 2.5 & 23 \\
11 & $\mathbf{b}[20]$ & 5 & 140 & 2.0 & $49(40)^{\mathrm{b}}$ \\
\hline
\end{tabular}

Reactions were performed using general procedure 1. a Performed under conventional heating using an oil bath.

b Pyridine was used as solvent. c Performed according to procedure 2.

\section{Procedure 1:}

2-Bromoresorcinol (50 mg, $0.27 \mathrm{mmol}, 1.0$ equiv.) was dissolved in the solvent stated (3.0 mL). Amine catalyst 5 ( 0.1 equiv. to 0.2 equiv.) and citral (1.0 to 2.5 equiv.) were added. The mixture was heated to the temperature stated in a sealed tube using microwave or conventional heating for the time stated. After cooling to room temperature, all volatile compounds were removed under reduced pressure. The residue was purified by MPLC (dry loading on Celite ${ }^{\circledR}$, cyclohexane/EtOAc $=100: 0$ to 0:100) to yield the title compound $\mathbf{6}$ as a yellow oil. For characterization data, see detailed procedure of 6 .

\section{Procedure 2:}

Citral (282 mg, $1.85 \mathrm{mmol}, 1.0$ equiv.) and piperidine ( $0.40 \mathrm{~mL}, 4.1 \mathrm{mmol}, 2.2$ equiv.) were dissolved in EtOAc $(3.5 \mathrm{~mL})$. Next, acetic anhydride $\left(0.41 \mathrm{~mL}, 4.3 \mathrm{mmol}, 2.3\right.$ equiv.) was added dropwise at $0{ }^{\circ} \mathrm{C}$ and the mixture was then heated to $90^{\circ} \mathrm{C}$ for $1 \mathrm{~h}$. After cooling to r.t., the mixture was added to a stirred solution of 2-bromoresorcinol (525 mg, $2.78 \mathrm{mmol}, 1.5$ equiv.) in toluene $(7.5 \mathrm{~mL})$ and heated to $150{ }^{\circ} \mathrm{C}$ in a sealed tube for $40 \mathrm{~h}$. The reaction mixture was quenched with $\mathrm{NaHCO}_{3}(50 \mathrm{~mL})$ and extracted with EtOAc $(3 \times 50 \mathrm{~mL})$. The combined organic layers were dried $\left(\mathrm{MgSO}_{4}\right)$, filtered and concentrated under reduced pressure. The residue was purified by MPLC (dry loading on Celite ${ }^{\circledR}$, cyclohexane/EtOAc = $100: 0$ to $0: 100$ ) to yield the title compound 6 (66 mg, $0.20 \mathrm{mmol}, 11 \%$ based on citral) as a yellow oil. For characterization data, see detailed procedure of 6 . 


\section{8-Bromo-2-methyl-2-(4-methylpent-3-en-1-yl)-2H-chromen-7-ol (6)}<smiles>Oc1cccc(O)c1Br</smiles>

3

$\mathrm{C}_{6} \mathrm{H}_{5} \mathrm{BrO}_{2}(189.01)$

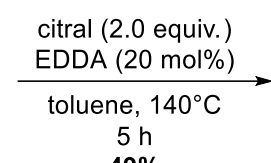

$49 \%$

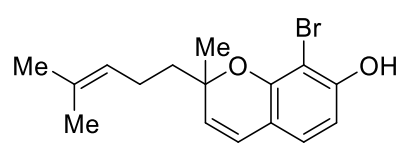

6

$\mathrm{C}_{16} \mathrm{H}_{19} \mathrm{BrO}_{2}(323.23)$

2-Bromoresorcinol (50 mg, $0.27 \mathrm{mmol}, 1.0$ equiv.) was dissolved in toluene (3.0 mL). Ethylenediamine$N, N$-diacetic acid (9.5 mg, $0.053 \mathrm{mmol}, 0.2$ equiv.) and citral (92 $\mu \mathrm{L}, 0.53 \mathrm{mmol}, 2.0$ equiv.) were added. The mixture was heated to $140^{\circ} \mathrm{C}$ in a sealed tube using microwave heating for $5 \mathrm{~h}$. After cooling to room temperature, all volatile compounds were removed under reduced pressure. The residue was purified by MPLC (dry loading on Celite $^{\circledR}$, cyclohexane/EtOAc $=100: 0$ to $0: 100$ ) to yield the title compound 6 as a yellow oil (42 mg, $0.13 \mathrm{mmol}, 49 \%$ ).

$\mathbf{R}_{\mathbf{f}}=0.26\left(n\right.$-hexane/EtOAc 95:5); ${ }^{1} \mathbf{H}$ NMR $\left(600 \mathrm{MHz}, \mathrm{CDCl}_{3}\right): \delta[\mathrm{ppm}]=6.82(\mathrm{~d}, J=8.2 \mathrm{~Hz}), 6.53(\mathrm{~d}$, $J=8.2 \mathrm{~Hz}$ ), $6.27(\mathrm{~d}, J=9.9 \mathrm{~Hz}, 1 \mathrm{H}), 5.45(\mathrm{~d}, J=9.9 \mathrm{~Hz}, 1 \mathrm{H}), 5.10(\mathrm{tt}, J=7.2,1.3 \mathrm{~Hz}, 1 \mathrm{H}), 2.15$ (d, $J=8.0 \mathrm{~Hz}, 1 \mathrm{H}), 2.12(\mathrm{~d}, J=7.7 \mathrm{~Hz}, 1 \mathrm{H}), 1.77-1.67(\mathrm{~m}, 2 \mathrm{H}), 1.66(\mathrm{~s}, 3 \mathrm{H}), 1.58(\mathrm{~s}, 3 \mathrm{H}), 1.44(\mathrm{~s}, 3 \mathrm{H})$; ${ }^{13} \mathrm{C} \mathrm{NMR}\left(151 \mathrm{MHz}, \mathrm{CDCl}_{3}\right): \delta[\mathrm{ppm}]=153.1,150.8,132.0,127.3,125.9,124.1,122.2,115.3,107.3$, 99.5, 80.4, 41.3, 26.6, 25.8, 22.7, 17.7; IR (neat): $\tilde{v}\left[\mathrm{~cm}^{-1}\right]=3506,3040,2969,2921,2856,1637,1604$, 1530, 1516, 1499, 1479, 1438, 1384, 1338, 1306, 1246, 1182, 1120, 1077, 1031, 983, 952, 915, 901, 810, 762, 715; HRMS (ESI): $\mathrm{m} / z$ calculated for $\mathrm{C}_{16} \mathrm{H}_{19} \mathrm{BrNaO}_{2}{ }^{+}\left([\mathrm{M}+\mathrm{Na}]^{+}\right): 345.0460$; found: 345.0461 .

2-Methyl-2-(4-methylpent-3-en-1-yl)-8-(trimethylsilyl)-2H-chromen-7-yl trifluoromethanesulfonate (7)
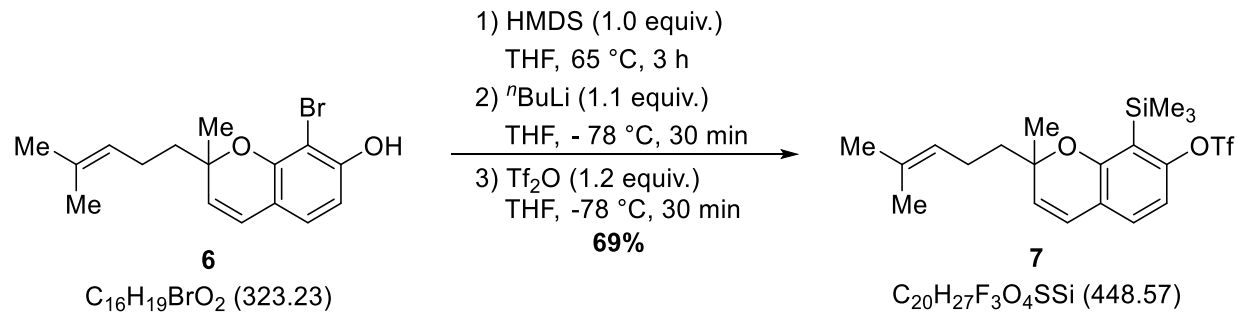

Compound 6 (100 mg, $0.309 \mathrm{mmol}, \quad 1.0$ equiv.) was dissolved in THF $(1.0 \mathrm{~mL})$ and hexamethyldisilazane ( $66 \mu \mathrm{L}, 0.31 \mathrm{mmol}, 1.0$ equiv.) was added. The mixture was heated to $65^{\circ} \mathrm{C}$ for $3 \mathrm{~h}$. After cooling to room temperature, all volatile compounds were removed under vacuum. The residue was dissolved in THF $(1.0 \mathrm{~mL})$ and $n$-butyllithium $(0.14 \mathrm{~mL}, 2.5 \mathrm{M}, 0.34 \mathrm{mmol}, 1.1$ equiv.) was added dropwise at $-78{ }^{\circ} \mathrm{C}$. After stirring at $-78{ }^{\circ} \mathrm{C}$ for $30 \mathrm{~min}$, trifluoromethanesulfonic anhydride $(62 \mu \mathrm{L}$, $0.37 \mathrm{mmol}, 1.2$ equiv.) was added and stirring was continued for $30 \mathrm{~min} . \mathrm{NaHCO}_{3}$ (sat. aq., $10 \mathrm{~mL}$ ) was added at $-78^{\circ} \mathrm{C}$ and the mixture was extracted with EtOAc ( $\left.3 \times 50 \mathrm{~mL}\right)$, dried $\left(\mathrm{Na}_{2} \mathrm{SO}_{4}\right)$, and concentrated under reduced pressure. The residue was purified via column chromatography $\left(\mathrm{SiO}_{2}, n\right.$-pentane/EtOAc 98:2) to yield the title compound 7 (95 $\mathrm{mg}, 0.21 \mathrm{mmol}, 69 \%)$ as a colorless oil.

$\mathbf{R}_{\mathbf{f}}=0.61\left(n\right.$-hexane/EtOAc 95:5); ${ }^{1} \mathbf{H} \mathbf{N M R}\left(500 \mathrm{MHz}, \mathrm{CDCl}_{3}\right): \delta[\mathrm{ppm}]=6.98(\mathrm{~d}, J=8.3 \mathrm{~Hz}, 1 \mathrm{H}), 6.81$ $(\mathrm{d}, J=8.3 \mathrm{~Hz}, 1 \mathrm{H}), 6.33(\mathrm{~d}, J=9.9 \mathrm{~Hz}, 1 \mathrm{H}), 5.58(\mathrm{~d}, J=9.9 \mathrm{~Hz}, 1 \mathrm{H}), 5.11$ (tdd, $J=5.7,2.8,1.4 \mathrm{~Hz}, 1 \mathrm{H}$ ), $2.23-2.01(\mathrm{~m}, 2 \mathrm{H}), 1.86(\mathrm{ddd}, J=13.9,11.6,5.2 \mathrm{~Hz}, 1 \mathrm{H}), 1.68(\mathrm{~s}, 3 \mathrm{H}), 1.67-1.61(\mathrm{~m}, 1 \mathrm{H}), 1.59(\mathrm{~s}$, 
$3 \mathrm{H}), 1.40(\mathrm{~s}, 3 \mathrm{H}), 0.40(\mathrm{~s}, 9 \mathrm{H}) ;{ }^{13} \mathrm{C}$ NMR $\left(126 \mathrm{MHz}, \mathrm{CDCl}_{3}\right) \delta[\mathrm{ppm}]=159.3,154.2,132.2,129.6,128.5$, 123.9, 122.3, 119.9, 119.2, 118.8 (q, $J=320.8 \mathrm{~Hz}), 112.4,80.2,42.1,27.1,25.8,23.4,17.8,1.2 ;{ }^{19} \mathbf{F}$ NMR $\left(471 \mathrm{MHz}, \mathrm{CDCl}_{3}\right) \delta[\mathrm{ppm}]=-72.8$; IR (neat): $\tilde{v}\left[\mathrm{~cm}^{-1}\right]=2970,2926,2858,1647,1576,1445$, 1407, 1370, 1341, 1286, 1247, 1206, 1163, 1139, 1076, 1016, 988, 923, 907, 840, 694, 668; HRMS (ESI): $\mathrm{m} / z$ calculated for $\mathrm{C}_{20} \mathrm{H}_{27} \mathrm{~F}_{3} \mathrm{NaO}_{4} \mathrm{SSi}^{+}\left([\mathrm{M}+\mathrm{Na}]^{+}\right): 471.1243$; found: 471.1245 .

2-Methoxy-N-(8-(4-methoxybenzoyl)-2-methyl-2-(4-methylpent-3-en-1-yl)-2H-chromen-7yl)acetamide (8) and 4-Methoxy-N-(8-(2-methoxyacetyl)-2-methyl-2-(4-methylpent-3-en-1-yl)-2Hchromen-7-yl)benzamide (9)
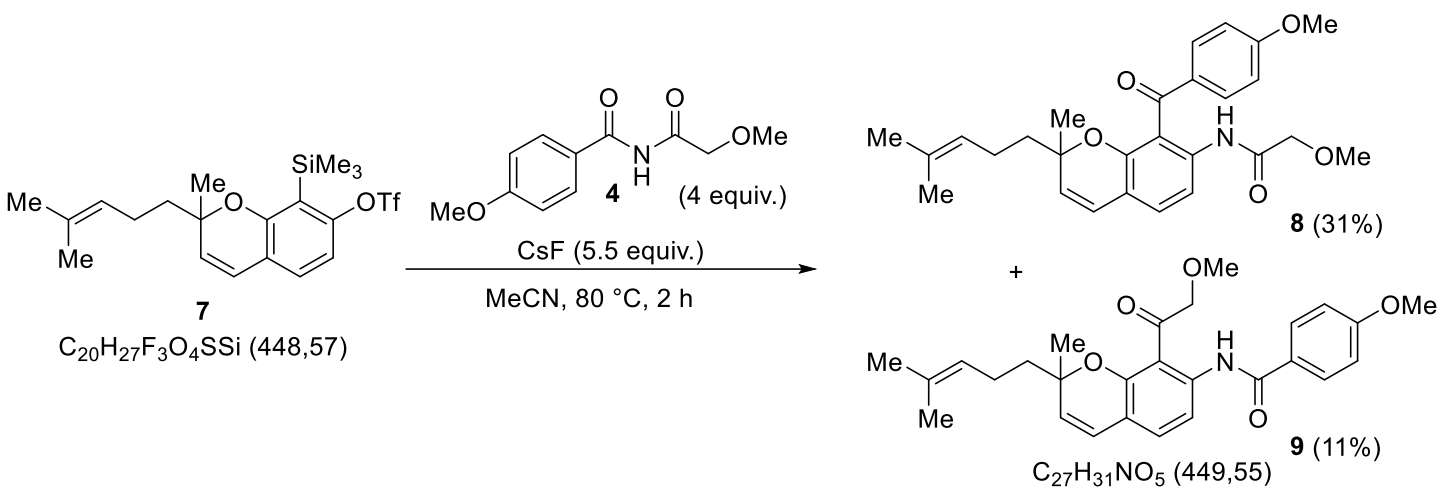

Cesium fluoride ( $93 \mathrm{mg}, 0.61 \mathrm{mmol}, 5.5$ equiv.) was dried at $10^{-3} \mathrm{mbar}$ and $500{ }^{\circ} \mathrm{C}$ for $5 \mathrm{~min}$. After cooling to room temperature, acetonitrile $(1.5 \mathrm{~mL})$ and imide $4(100 \mathrm{mg}, 0.45 \mathrm{mmol}, 4.0$ equiv.) were added. After adding aryne precursor 7 ( $50 \mathrm{mg}, 0.11 \mathrm{mmol}, 1.0$ equiv.), the mixture was heated to $80^{\circ} \mathrm{C}$ for $2 \mathrm{~h}$. After cooling to room temperature, the solvent was removed under reduced pressure and the residue was purified by MPLC (dry loading on Celite ${ }^{\circledR}$, cyclohexane/EtOAc 100:0 to 0:100), yielding 8 (15 mg, $34 \mu \mathrm{mol}, 31 \%$ ) and 9 ( $5.7 \mathrm{mg}, 13 \mu \mathrm{mol}, 11 \%$ ) as yellow oils. The thus obtained racemic mixture of 8 can be separated into its enantiomers by chiral HPLC (chiralPAK ${ }^{\circledR} I A, n$-hexane/i-PrOH 95:5, flow: $18 \mathrm{~mL} \cdot \mathrm{min}^{-1}$; 90 bar; ee $\geq 99 \%$ [HPLC]).

$(+)-8:[\alpha]_{\mathrm{D}}^{24}=+128.6^{\circ}\left(\mathrm{c}=0.22, \mathrm{CHCl}_{3}\right) ;(-)-8:[\alpha]_{\mathrm{D}}^{24}=-146.3^{\circ}\left(\mathrm{c}=0.19, \mathrm{CHCl}_{3}\right)$

$\mathbf{R}_{\mathbf{f}}=0.22(n$-hexane/EtOAc $=4: 1) ;{ }^{1} \mathbf{H}$ NMR $\left(700 \mathrm{MHz}, \mathrm{CDCl}_{3}\right) \delta[\mathrm{ppm}]=9.25(\mathrm{~s}, 1 \mathrm{H}), 7.83-7.78(\mathrm{~m}$, $3 \mathrm{H}), 7.06(\mathrm{~d}, J=8.3 \mathrm{~Hz}, 1 \mathrm{H}), 6.91-6.87(\mathrm{~m}, 2 \mathrm{H}), 6.33(\mathrm{~d}, J=9.9 \mathrm{~Hz}, 1 \mathrm{H}), 5.46(\mathrm{~d}, J=10.0 \mathrm{~Hz}, 1 \mathrm{H})$, 4.90 (ddt, $J=7.1,5.7,1.4 \mathrm{~Hz}, 1 \mathrm{H}), 3.90(\mathrm{~d}, J=0.6 \mathrm{~Hz}, 2 \mathrm{H}), 3.85(\mathrm{~s}, 3 \mathrm{H}), 3.37(\mathrm{~s}, 3 \mathrm{H}), 1.83-1.76(\mathrm{~m}$, $1 \mathrm{H}), 1.75-1.68(\mathrm{~m}, 1 \mathrm{H}), 1.61(\mathrm{~d}, J=1.3 \mathrm{~Hz}, 3 \mathrm{H}), 1.45(\mathrm{~d}, J=1.4 \mathrm{~Hz}, 3 \mathrm{H}), 1.43-1.38(\mathrm{~m}, 2 \mathrm{H}), 1.12(\mathrm{~s}$, $3 \mathrm{H}) ;{ }^{13} \mathrm{C}$ NMR $\left(176 \mathrm{MHz}, \mathrm{CDCl}_{3}\right) \delta[\mathrm{ppm}]=195.3,168.3,163.8,151.6,135.9,132.0,131.9,131.8,129.0$, 128.7, 124.0, 122.1, 118.1, 117.7, 114.1, 113.6, 79.7, 72.3, 59.6, 55.6, 41.5, 26.5, 25.7, 22.5, 17.7; IR (neat): $\tilde{v}\left[\mathrm{~cm}^{-1}\right]=3356,2966,2924,2852,1717,1700,1683,1669,1662,1653,1647,1635,1596,1577$, 1540, 1533, 1521, 1517, 1507, 1457, 1430, 1418, 1399, 1307, 1285, 1257, 1192, 1170, 1153, 1112, 1083, 1029, 987, 947, 890, 843, 793, 762, 741, 720; HRMS (ESI): $m / z$ calculated for $\mathrm{C}_{27} \mathrm{H}_{31} \mathrm{NNaO}_{5}{ }^{+}$ $\left([\mathrm{M}+\mathrm{Na}]^{+}\right):$472.2094; found: 472.2091 .

9:

$\mathbf{R}_{\mathbf{f}}=0.35$ ( $n$-hexane/EtOAc $\left.=4: 1\right) ;{ }^{1} \mathbf{H}$ NMR $\left(700 \mathrm{MHz}, \mathrm{CDCl}_{3}\right) \delta[\mathrm{ppm}]=12.37(\mathrm{~s}, 1 \mathrm{H}), 8.41(\mathrm{~d}, \mathrm{~J}=$ $8.5 \mathrm{~Hz}, 1 \mathrm{H}), 8.06-8.02(\mathrm{~m}, 2 \mathrm{H}), 7.17(\mathrm{~d}, J=8.5 \mathrm{~Hz}, 1 \mathrm{H}), 7.02-6.96(\mathrm{~m}, 2 \mathrm{H}), 6.37(\mathrm{~d}, J=9.9 \mathrm{~Hz}, 1 \mathrm{H})$, 
$5.56(\mathrm{~d}, J=9.9 \mathrm{~Hz}, 1 \mathrm{H}), 5.12-5.08(\mathrm{~m}, 1 \mathrm{H}), 4.74(\mathrm{~d}, J=18.0 \mathrm{~Hz}, 1 \mathrm{H}), 4.71(\mathrm{~d}, J=18.0 \mathrm{~Hz}, 1 \mathrm{H}), 3.88$ (s, 3H), 3.51 (s, 3H), $2.21-2.07(\mathrm{~m}, 2 \mathrm{H}), 1.90$ (ddd, $J=14.0,11.1,5.4 \mathrm{~Hz}, 1 \mathrm{H}), 1.73$ (ddd, $J=14.1$, 11.3, $5.2 \mathrm{~Hz}, 1 \mathrm{H}), 1.67(\mathrm{~d}, J=1.4 \mathrm{~Hz}, 3 \mathrm{H}), 1.57(\mathrm{~d}, J=1.3 \mathrm{~Hz}, 3 \mathrm{H}), 1.49(\mathrm{~s}, 3 \mathrm{H}) ;{ }^{13} \mathrm{C}$ NMR $(176 \mathrm{MHz}$, $\left.\mathrm{CDCl}_{3}\right) \delta[\mathrm{ppm}]=202.1,165.6,162.8,155.6,141.9,132.5,132.5,129.6,127.4,127.2,123.5,122.7$, $116.4,114.2,113.3,111.9,81.6,80.3,59.5,55.6,42.0,27.3,25.8,23.3,17.8$; IR (neat): $\tilde{v}\left[\mathrm{~cm}^{-1}\right]=2957$, 2924, 2853, 1680, 1650, 1596, 1507, 1454, 1408, 1376, 1306, 1254, 1181, 1124, 1081, 1030, 925, 842, 756; HRMS (ESI): $\mathrm{m} / z$ calculated for $\mathrm{C}_{27} \mathrm{H}_{31} \mathrm{NNaO}_{5}{ }^{+}\left([\mathrm{M}+\mathrm{Na}]^{+}\right): 472.2094$; found: 472.2080 .

\section{Yaequinolone J1 (1) and Yaequinolone J2 (2)}

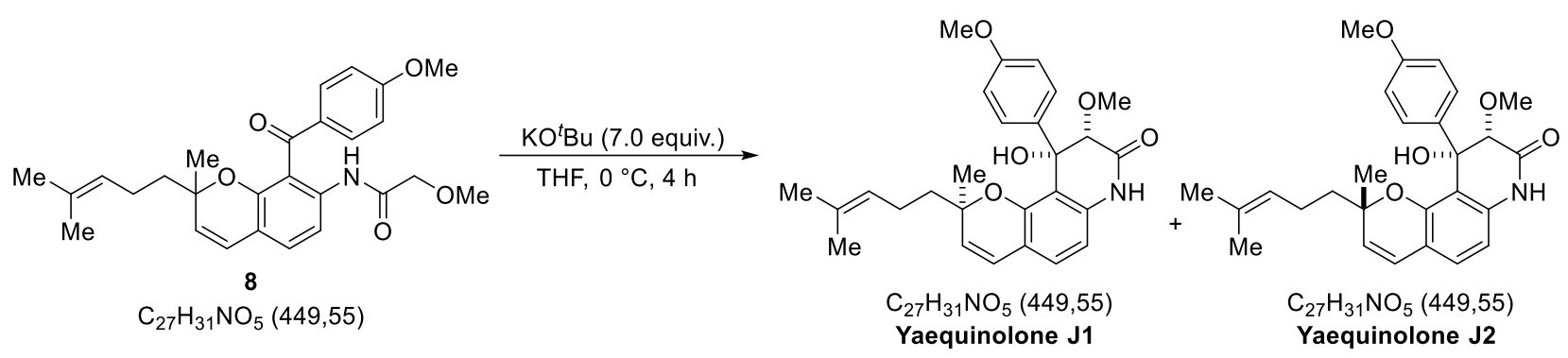

Compound 8 ( $25 \mathrm{mg}, 56 \mu \mathrm{mol}, 1.0$ equiv.) was dissolved in anhydrous THF ( $3.0 \mathrm{~mL}$ ) and a solution of $\mathrm{KO} B \mathrm{Bu}\left(0.78 \mathrm{~mL}, 0.39 \mathrm{mmol}, 0.5 \mathrm{M}\right.$ in THF, 7.0 equiv.) was added at $0{ }^{\circ} \mathrm{C}$. The mixture was stirred at 0 ${ }^{\circ} \mathrm{C}$ until complete consumption of starting material was observed via TLC (4 h). The reaction was quenched by addition of water and extracted with EtOAc $(3 \times 50 \mathrm{~mL})$. The combined organic extracts were dried $\left(\mathrm{MgSO}_{4}\right)$ and concentrated under reduced pressure. The residue was purified via column chromatography $\left(\mathrm{SiO}_{2}, \mathrm{CH}_{2} \mathrm{Cl}_{2} / \mathrm{MeOH} 97: 3\right)$ to yield a mixture of yaequinolone $\mathrm{J} 1$ and $\mathrm{J} 2$ (18 $\mathrm{mg}$, $0.41 \mathrm{mmol}, d . r .=1: 1.4$ ['H NMR], 74\%) as a colorless oil. The two diastereomers were separated by preparative $\mathrm{TLC}\left(\mathrm{SiO}_{2}, \mathrm{CH}_{2} \mathrm{Cl}_{2} / \mathrm{MeOH} 98: 2\right)$, to yield pure yaequinolone $\mathrm{J} 1(7.3 \mathrm{mg}, 16 \mu \mathrm{mol}, 29 \%)$ and yaequinolone $\mathrm{J} 2$ (10 mg, $22 \mu \mathrm{mol}, 40 \%$ ) as colorless oils.

According to the synthesis of racemic yaequinolones $\mathrm{J} 1$ and $\mathrm{J} 2$, (-)-yaequinolone $\mathrm{J} 1$ (2.5 mg, $5.6 \mu \mathrm{mol}$, $30 \%)$ was obtained starting from (-)-8 $(8.2 \mathrm{mg}, 18 \mu \mathrm{mol})$ and $\mathrm{KO}$ 'Bu $(0.26 \mathrm{~mL}, 0.13 \mathrm{mmol}, 0.5 \mathrm{M}$ in THF). (+)-yaequinolone $\mathrm{J} 2(2.1 \mathrm{mg}, 4.7 \mu \mathrm{mol}, 40 \%)$ was obtained starting from (+)-8 (5.3 mg, $12 \mu \mathrm{mol})$ and $\mathrm{KO} B \mathrm{Bu}(0.17 \mathrm{~mL}, 83 \mu \mathrm{mol}, 0.5 \mathrm{M}$ in THF).

\section{(-)-Yaequinolone J1:}

$[\alpha]_{\mathrm{D}}^{21}=-61.9^{\circ}(\mathrm{c}=0.21, \mathrm{EtOH})\left[\mathrm{Lit}^{2 \mathrm{~b}}[\alpha]_{\mathrm{D}}^{23}=-65.6^{\circ}(\mathrm{c}=0.1, \mathrm{EtOH})\right] ; \mathbf{R}_{\mathrm{f}}=0.37\left(\mathrm{CH}_{2} \mathrm{Cl}_{2} / \mathrm{MeOH} 95: 5\right) ;$ ${ }^{1} \mathrm{H}$ NMR $\left(700 \mathrm{MHz}, \mathrm{CDCl}_{3}\right) \delta[\mathrm{ppm}]=7.67(\mathrm{~s}, 1 \mathrm{H}), 7.21-7.18(\mathrm{~m}, 2 \mathrm{H}), 6.90(\mathrm{~d}, J=7.9 \mathrm{~Hz}, 1 \mathrm{H}), 6.80-$ $6.76(\mathrm{~m}, 2 \mathrm{H}), 6.34(\mathrm{~d}, J=8.0 \mathrm{~Hz}, 1 \mathrm{H}), 6.28(\mathrm{~d}, J=10.0 \mathrm{~Hz}, 1 \mathrm{H}), 5.44(\mathrm{~d}, J=10.0 \mathrm{~Hz}, 1 \mathrm{H}), 5.34(\mathrm{~s}, 1 \mathrm{H})$, 4.72 (tdd, $J=6.9,3.0,1.5 \mathrm{~Hz}, 1 \mathrm{H}$ ), 3.80 (d, $J=1.4 \mathrm{~Hz}, 1 \mathrm{H}$ ), $3.74(\mathrm{~s}, 3 \mathrm{H}), 3.58(\mathrm{~s}, 3 \mathrm{H}), 1.73-1.66$ (m, $1 \mathrm{H}), 1.60-1.52(\mathrm{~m}, 5 \mathrm{H}), 1.43-1.39(\mathrm{~m}, 4 \mathrm{H}), 1.32(\mathrm{~s}, 3 \mathrm{H}) ;{ }^{13} \mathrm{C} \mathrm{NMR}\left(176 \mathrm{MHz}, \mathrm{CDCl}_{3}\right) \delta[\mathrm{ppm}]=167.5$, $159.7,152.5,136.4,133.8,131.9,127.9,127.5,127.0,123.6,122.2,117.8,114.4,114.1,108.1,85.1$, 80.8, 78.0, 59.6, 55.3, 41.1, 26.7, 25.7, 22.1, 17.5; IR (neat): $\tilde{v}\left[\mathrm{~cm}^{-1}\right]=3495,3225,2965,2929,2857$, 2836, 1694, 1606, 1510, 1492, 1377, 1252, 1176, 1102, 1046, 827, 742, $716 \mathrm{~cm}^{-1}$; HRMS (ESI): $\mathrm{m} / \mathrm{z}$ calculated for $\mathrm{C}_{27} \mathrm{H}_{31} \mathrm{NNaO}_{5}{ }^{+}\left([\mathrm{M}+\mathrm{Na}]^{+}\right)$: 472.2094; found: 472.2092. The spektroscopic data matches those reported for (-)-Yaequinolone $\mathrm{J} 1 .^{2}$ 


\section{(+)-Yaequinolone J2:}

$[\alpha]_{\mathrm{D}}^{21}=+176.5^{\circ}(\mathrm{c}=0.18, \mathrm{EtOH})\left[\mathrm{Lit}: 2 \mathrm{~b}[\alpha]_{\mathrm{D}}^{23}=+187.1^{\circ}(\mathrm{c}=0.1, \mathrm{EtOH})\right] ; \mathbf{R}_{\mathbf{f}}=0.39\left(\mathrm{CH}_{2} \mathrm{Cl}_{2} / \mathrm{MeOH} 95: 5\right) ;$ ${ }^{1} \mathrm{H}$ NMR $\left(700 \mathrm{MHz}, \mathrm{CDCl}_{3}\right) \delta[\mathrm{ppm}]=7.81(\mathrm{~s}, 1 \mathrm{H}), 7.21-7.18(\mathrm{~m}, 2 \mathrm{H}), 6.91(\mathrm{~d}, J=8.0 \mathrm{~Hz}, 1 \mathrm{H}), 6.81-$ $6.77(\mathrm{~m}, 2 \mathrm{H}), 6.36(\mathrm{~d}, J=8.0 \mathrm{~Hz}, 1 \mathrm{H}), 6.31(\mathrm{~d}, J=10.0 \mathrm{~Hz}, 1 \mathrm{H}), 5.50(\mathrm{~d}, J=9.9 \mathrm{~Hz}, 1 \mathrm{H}), 5.20(\mathrm{~s}, 1 \mathrm{H})$, 5.04 (tdd, $J=5.7,2.8,1.4 \mathrm{~Hz}, 1 \mathrm{H}$ ), $3.83(\mathrm{~d}, J=1.4 \mathrm{~Hz}, 1 \mathrm{H}), 3.76(\mathrm{~s}, 3 \mathrm{H}), 3.58(\mathrm{~s}, 3 \mathrm{H}), 2.09$ (dq, $J=$ 12.4, $6.6 \mathrm{~Hz}, 1 \mathrm{H}), 2.04-1.97(\mathrm{~m}, 1 \mathrm{H}), 1.70-1.60(\mathrm{~m}, 5 \mathrm{H}), 1.57(\mathrm{~s}, 3 \mathrm{H}), 0.95(\mathrm{~s}, 3 \mathrm{H}) ;{ }^{13} \mathrm{C}$ NMR $\left(176 \mathrm{MHz}, \mathrm{CDCl}_{3}\right) \delta[\mathrm{ppm}]=167.7,159.6,152.6,136.3,134.2,132.4,128.3,127.5,127.0,123.7,122.6$, $118.3,114.9,113.9,108.2,85.0,80.5,78.0,59.7,55.4,41.4,25.8,25.7,23.1,17.8$; IR (neat): $\tilde{v}$ [cm $\left.{ }^{-1}\right]=$ 3507, 3235, 3062, 3043, 2967, 2925, 2853, 2836, 1694, 1641, 1605, 1510, 1491, 1462, 1377, 1303, $1252,1222,1175,1104,1081,1046,998,916,883,830,753,734$; HRMS (ESI): $m / z$ calculated for $\mathrm{C}_{27} \mathrm{H}_{31} \mathrm{NNaO}_{5}{ }^{+}\left([\mathrm{M}+\mathrm{Na}]^{+}\right): 472.2094$; found: 472.2093. The spektroscopic data matches those reported for (+)-Yaequinolone J2.2 
${ }^{1} \mathrm{H}$ and ${ }^{13} \mathrm{C}$ NMR comparisons $\left(\mathrm{CDCl}_{3}\right)$

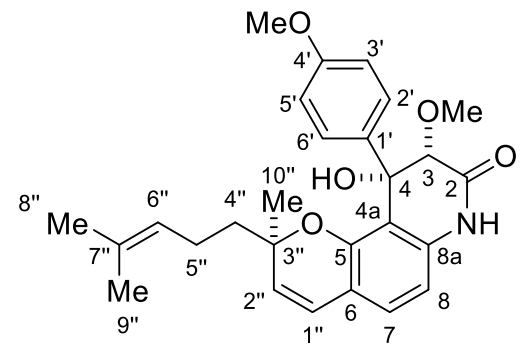

Yaequinolone J1

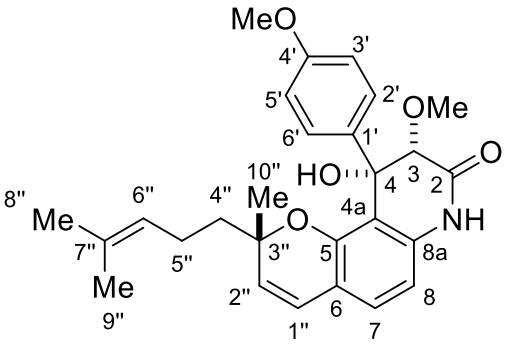

Yaequinolone J2

\begin{tabular}{|c|c|c|c|c|c|}
\hline $\begin{array}{c}{ }^{1} \text { H NMR } \\
\text { Data }\end{array}$ & $\begin{array}{l}\text { Synthetic } \\
(700 \mathrm{MHz})\end{array}$ & $\begin{array}{l}\text { Natural }^{2 b} \\
(600 \mathrm{MHz})\end{array}$ & $\begin{array}{c}{ }^{1} \mathrm{H} \text { NMR } \\
\text { Data }\end{array}$ & $\begin{array}{l}\text { Synthetic } \\
(700 \mathrm{MHz})\end{array}$ & $\begin{array}{c}\text { Natural }^{2 b} \\
(600 \mathrm{MHz})\end{array}$ \\
\hline Position & \multicolumn{2}{|c|}{$\delta \mathrm{H}(\mathrm{J}$ in $\mathrm{Hz})$} & Position & \multicolumn{2}{|c|}{$\delta \mathrm{H}(\mathrm{J}$ in $\mathrm{Hz})$} \\
\hline $1-\mathrm{NH}$ & 7.67, br s & 7.40, br s & 1-NH & 7.81, br s & 7.49, br s \\
\hline 3 & $3.80, \mathrm{~d}(1.4)$ & $3.80, d(1.1)$ & 3 & 3.83, d (1.4) & $3.83, d(0.9)$ \\
\hline 7 & $6.90, \mathrm{~d}(7.9)$ & $6.90, d(8.0)$ & 7 & $6.91, d(8.0)$ & $6.93, d(8.0)$ \\
\hline 8 & $6.34(8.0)$ & $6.34, d(8.0)$ & 8 & $6.36, d(8.0)$ & $6.36, d(8.0)$ \\
\hline $2^{\prime}, 6^{\prime}$ & 7.19, m & $7.19, \mathrm{~d}(8.4)$ & $2^{\prime}, 6^{\prime}$ & 7.19, m & $7.19, d(8.8)$ \\
\hline $3^{\prime}, 5^{\prime}$ & $6.78, m$ & $6.78, d(8.4)$ & $3^{\prime}, 5^{\prime}$ & 6.79, m & $6.79, d(8.8)$ \\
\hline $1 "$ & $6.28, d(10.0)$ & $6.28, d(9.6)$ & $1 "$ & $6.31, d(10.0)$ & $6.31, d(9.7)$ \\
\hline 2" & $5.44, d(10.0)$ & $5.44, d(9.6)$ & 2" & $5.50, d(9.9)$ & $5.50, d(9.7)$ \\
\hline $4 "$ & $1.41, \mathrm{~m}$ & $1.40, \mathrm{~m}$ & $4 "$ & $\begin{array}{l}1.65, \mathrm{~m} \\
1.65, \mathrm{~m}\end{array}$ & $\begin{array}{l}1.67, \mathrm{~m} \\
1.62, \mathrm{~m}\end{array}$ \\
\hline $5 "$ & $\begin{array}{l}1.69, \mathrm{~m} \\
1.56, \mathrm{~m}\end{array}$ & $\begin{array}{l}1.69, \mathrm{~m} \\
1.56, \mathrm{~m}\end{array}$ & $5 "$ & $\begin{array}{c}2.09, \mathrm{dq}(12.4, \\
6.6)\end{array}$ & $2.09, \mathrm{~m}$ \\
\hline 6" & $\begin{array}{c}4.72, \text { tdd }(6.9 \\
3.0,1.5)\end{array}$ & $4.71, \mathrm{t}(7.1)$ & 6" & $\begin{array}{c}2.00, \mathrm{~m} \\
5.04, \mathrm{tdd}(5.7 \\
2.8,1.4)\end{array}$ & $\begin{array}{c}2.01, \mathrm{~m} \\
5.04, \mathrm{t}(7.3)\end{array}$ \\
\hline $8 "$ & $1.56, \mathrm{~m}$ & $1.56, \mathrm{~s}$ & 8" & $1.65, \mathrm{~m}$ & $1.66, \mathrm{~s}$ \\
\hline $9 "$ & $1.32, \mathrm{~s}$ & $1.31, \mathrm{~s}$ & $9 "$ & $1.57, \mathrm{~s}$ & $1.57, \mathrm{~s}$ \\
\hline $10^{\prime \prime}$ & $1.41, \mathrm{~m}$ & $1.41, \mathrm{~s}$ & $10^{\prime \prime}$ & $0.95, \mathrm{~s}$ & $0.95, \mathrm{~s}$ \\
\hline 3-OMe & $3.58, \mathrm{~s}$ & $3.58, \mathrm{~s}$ & 3-OMe & $3.58, \mathrm{~s}$ & $3.58, \mathrm{~s}$ \\
\hline 4'-OMe & $3.74, \mathrm{~s}$ & $3.74, \mathrm{~s}$ & 4'-OMe & $3.76, \mathrm{~s}$ & $3.77, \mathrm{~s}$ \\
\hline $4-\mathrm{OH}$ & $5.34, \mathrm{~s}$ & $5.36, \mathrm{~s}$ & $4-\mathrm{OH}$ & $5.20, \mathrm{~s}$ & $5.22, \mathrm{~s}$ \\
\hline
\end{tabular}




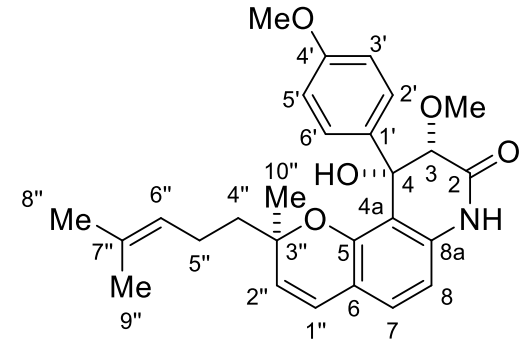

Yaequinolone J1

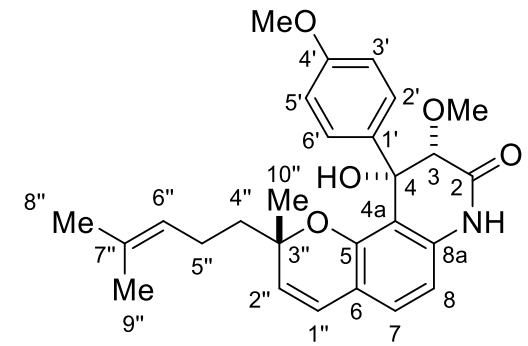

Yaequinolone J2

\begin{tabular}{|c|c|c|c|c|c|}
\hline $\begin{array}{c}{ }^{13} \text { C NMR } \\
\text { Data }\end{array}$ & $\begin{array}{l}\text { Synthetic } \\
\text { (176 MHz) }\end{array}$ & $\begin{array}{l}\text { Natural }^{2 b} \\
(150 \mathrm{MHz})\end{array}$ & $\begin{array}{c}{ }^{13} \text { C NMR } \\
\text { Data }\end{array}$ & $\begin{array}{l}\text { Synthetic } \\
\text { (176 MHz) }\end{array}$ & $\begin{array}{l}\text { Natural }^{2 b} \\
(150 \mathrm{MHz})\end{array}$ \\
\hline Position & \multicolumn{2}{|c|}{$\delta \mathrm{H}(\mathrm{J}$ in $\mathrm{Hz})$} & Position & \multicolumn{2}{|c|}{$\delta \mathrm{H}(\mathrm{J}$ in $\mathrm{Hz})$} \\
\hline 2 & 167.5 & 167.2 & 2 & 167.7 & 167.4 \\
\hline 3 & 85.1 & 85.1 & 3 & 85.0 & 85.0 \\
\hline 4 & 78.0 & 78.1 & 4 & 78.0 & 78.0 \\
\hline $4 a$ & 114.4 & 114.3 & $4 a$ & 114.9 & 114.6 \\
\hline 5 & 152.5 & 152.5 & 5 & 152.6 & 152.6 \\
\hline 6 & 117.8 & 118.0 & 6 & 118.3 & 118.5 \\
\hline 7 & 127.0 & 127.2 & 7 & 127.0 & 127.1 \\
\hline 8 & 108.1 & 108.1 & 8 & 108.2 & 108.2 \\
\hline $8 a$ & 136.4 & 136.2 & $8 a$ & 136.3 & 136.2 \\
\hline $1^{\prime}$ & 133.8 & 133.7 & $1^{\prime}$ & 134.2 & 134.1 \\
\hline $2^{\prime}, 6^{\prime}$ & 127.5 & 127.5 & $2^{\prime}, 6^{\prime}$ & 127.5 & 127.4 \\
\hline $3^{\prime}, 5^{\prime}$ & 114.1 & 114.2 & $3^{\prime}, 5^{\prime}$ & 113.9 & 113.9 \\
\hline $4^{\prime}$ & 159.7 & 159.8 & $4^{\prime}$ & 159.6 & 159.8 \\
\hline $1 "$ & 122.2 & 122.2 & $1 "$ & 122.6 & 122.5 \\
\hline $2 "$ & 127.9 & 128.0 & $2 "$ & 128.3 & 128.4 \\
\hline $3 "$ & 80.8 & 80.8 & 3" & 80.5 & 80.5 \\
\hline $4 "$ & 41.1 & 41.1 & $4 "$ & 41.4 & 41.3 \\
\hline $5 "$ & 22.1 & 22.2 & $5 "$ & 23.1 & 23.0 \\
\hline 6" & 123.6 & 123.7 & 6" & 123.7 & 123.8 \\
\hline 7" & 131.9 & 131.9 & 7" & 132.4 & 132.4 \\
\hline 8" & 25.7 & 25.6 & $8 "$ & 25.8 & 25.8 \\
\hline 9" & 17.5 & 17.4 & 9" & 17.8 & 17.7 \\
\hline $10 "$ & 26.7 & 26.7 & $10 "$ & 25.7 & 25.6 \\
\hline 3-OMe & 59.6 & 59.5 & 3-OMe & 59.7 & 59.7 \\
\hline 4'-OMe & 55.3 & 55.3 & 4'-OMe & 55.4 & 55.4 \\
\hline
\end{tabular}




\section{References}

(1) Schwan, J.; Kleoff, M.; Hartmayer, B.; Heretsch, P.; Christmann, M. Synthesis of Quinolinone Alkaloids via Aryne Insertions into Unsymmetric Imides in Flow. Org. Lett. 2018, 20, 7661-7664.

(2) (a) Vece, V.; Jakkepally, S.; Hanessian, S. Total Synthesis and Absolute Stereochemical Assignment of the Insecticidal Methabolites Yaequinolones J1 and J2. Org. Lett. 2018, 20, 4277-4280. (b) Uchida, R.; Imasato, R.; Shiomi, K.; Tomoda, H.; Ōmura, S. Yaequinolones J1 and J2, Novel Insecticidal Antibiotics from Penicillium sp. FKI-2140. Org. Lett. 2005, 7, 57015704. 


\section{Appendix}

HPLC chromatogram of rac-8 (ChiralPAK IA, 10\% IPA/hexane, 34 bar, $0.8 \mathrm{~mL} / \mathrm{min}$ ):

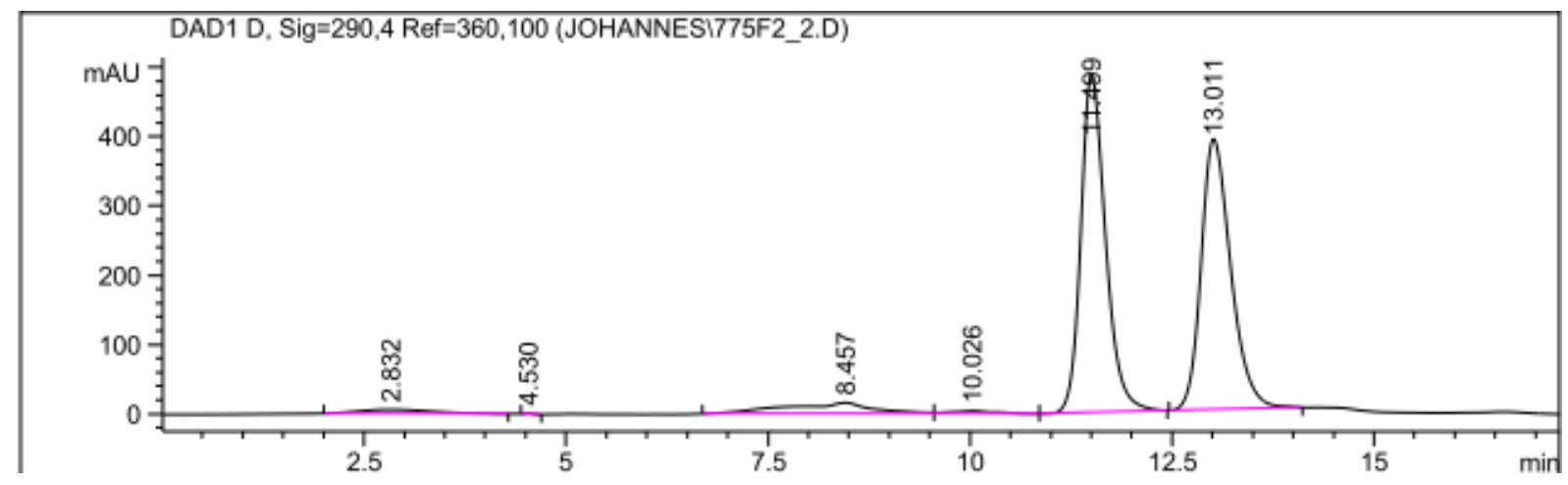

Signal 2: DAD1 D, Sig=290, 4 Ref $=360,100$

\begin{tabular}{|c|c|c|c|c|c|c|}
\hline $\begin{array}{c}\text { Peak } \\
\#\end{array}$ & $\begin{array}{c}\text { RetTime } \\
\text { [min] }\end{array}$ & Type & $\begin{array}{l}\text { Width } \\
\text { [min] }\end{array}$ & $\begin{array}{c}\text { Area } \\
{[\mathrm{mAU} * \mathrm{~s}]}\end{array}$ & $\begin{array}{l}\text { Height } \\
\text { [MAU] }\end{array}$ & $\begin{array}{c}\text { Area } \\
\frac{\circ}{b}\end{array}$ \\
\hline & & & & $=-$ & --- & 年 \\
\hline 1 & 2.832 & BB & 0.7588 & 383.23703 & 5.98713 & 1.7278 \\
\hline 2 & 4.530 & BB & 0.0855 & 7.06715 & 1.34046 & 0.0319 \\
\hline 3 & 8.457 & BV & 0.9825 & 1241.70569 & 16.05212 & 5.5982 \\
\hline 4 & 10.026 & vV & 0.5045 & 160.19423 & 4.16720 & 0.7222 \\
\hline 5 & 11.499 & VB & 0.3243 & $1.03764 \mathrm{e} 4$ & 487.50809 & 46.7814 \\
\hline 6 & 13.011 & BB & 0.3911 & $1.00120 \mathrm{e} 4$ & 390.47000 & 45.1385 \\
\hline
\end{tabular}

HPLC chromatogram of (+)-8 (ChiralPAK IA, 10\% IPA/hexane, $34 \mathrm{bar}, 0.8 \mathrm{~mL} / \mathrm{min}$ ):

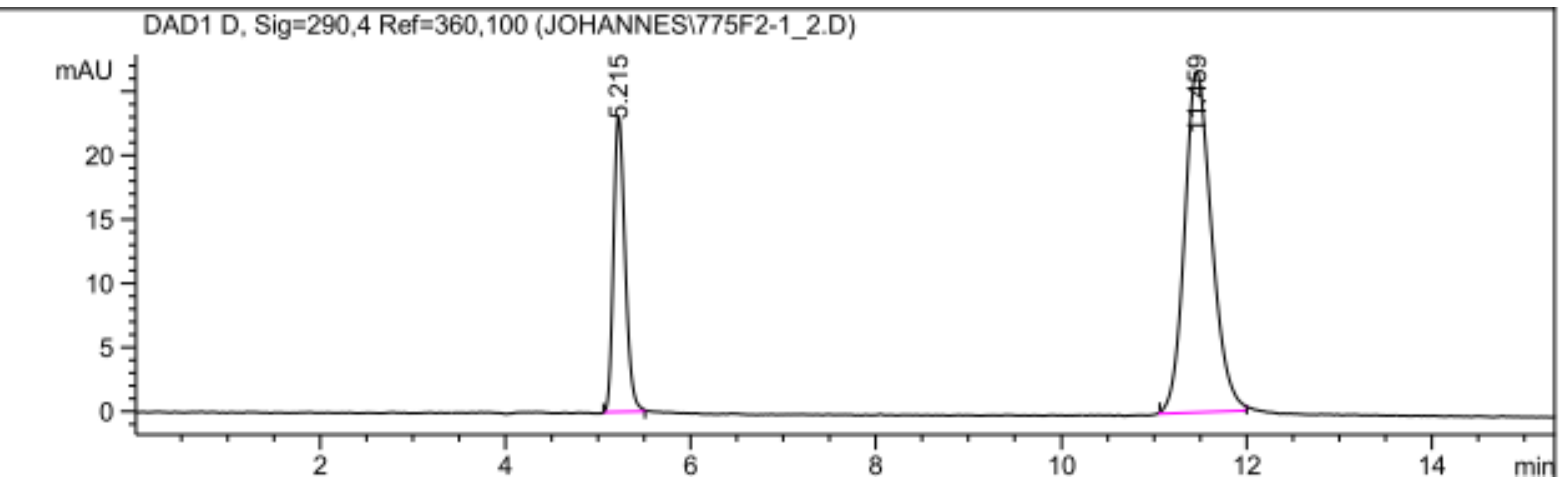

Signal 2: DAD1 D, Sig=290, 4 Ref $=360,100$

\begin{tabular}{|c|c|c|c|c|c|c|}
\hline $\begin{array}{c}\text { Peak } \\
\#\end{array}$ & $\begin{array}{c}\text { RetTime } \\
\text { [min] }\end{array}$ & Type & $\begin{array}{l}\text { Width } \\
\text { [min] }\end{array}$ & $\begin{array}{c}\text { Area } \\
{[\mathrm{mAU} * \mathrm{~s}]}\end{array}$ & $\begin{array}{l}\text { Height } \\
\text { [MAU] }\end{array}$ & $\begin{array}{c}\text { Area } \\
\frac{2}{5}\end{array}$ \\
\hline & & & & (20) & & \\
\hline 1 & 5.215 & BB & 0.1357 & 201.69472 & 23.14871 & 27.1269 \\
\hline 2 & 11.459 & BB & 0.3073 & 541.82794 & 26.64318 & 72.8731 \\
\hline
\end{tabular}


HPLC chromatogram of (-)-8 (ChiralPAK IA, 10\% IPA/hexane, $34 \mathrm{bar}, 0.8 \mathrm{~mL} / \mathrm{min}$ ):

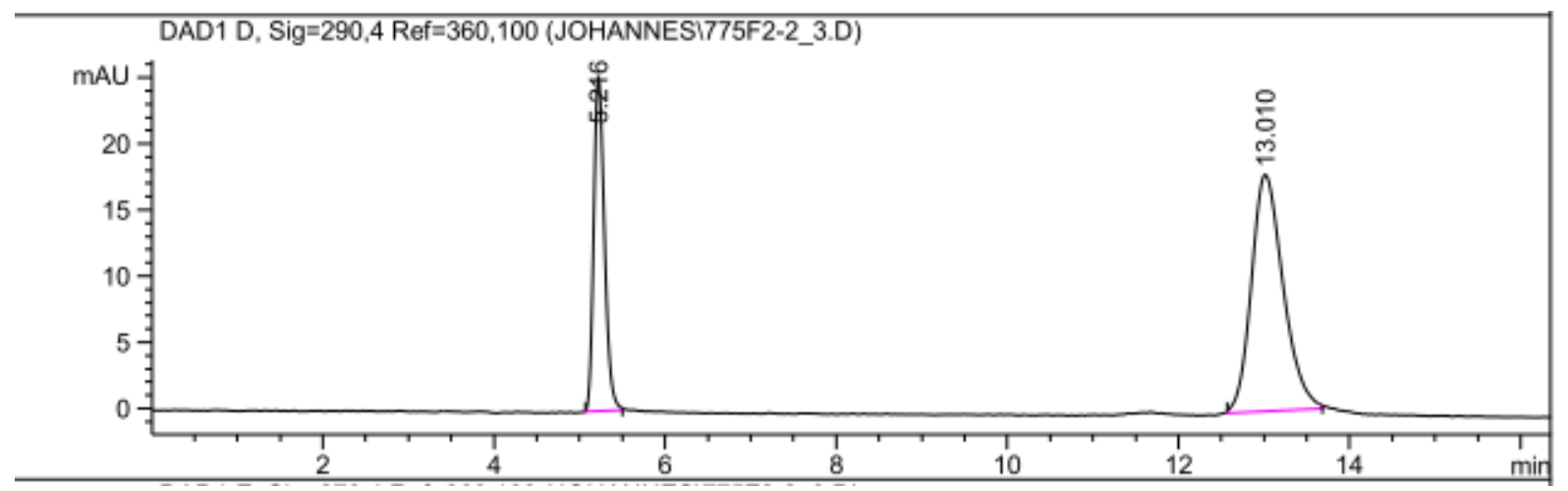

Signal 2: DAD1 D, Sig=290,4 $\operatorname{Ref}=360,100$

\begin{tabular}{|c|c|c|c|c|c|c|}
\hline $\begin{array}{c}\text { Peak } \\
\#\end{array}$ & $\begin{array}{c}\text { RetTime } \\
\text { [min] }\end{array}$ & Type & $\begin{array}{l}\text { Width } \\
\text { [min] }\end{array}$ & $\begin{array}{c}\text { Area } \\
{\left[\mathrm{mAU}^{\star} \mathrm{s}\right]}\end{array}$ & $\begin{array}{l}\text { Height } \\
\text { [mAU] }\end{array}$ & $\begin{array}{c}\text { Area } \\
\frac{8}{\delta}\end{array}$ \\
\hline 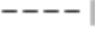 & 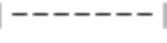 & & . & ---- & 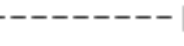 & --- \\
\hline 1 & 5.216 & BB & 0.1357 & 220.38380 & 25.29259 & 32.1903 \\
\hline 2 & 13.010 & BB & 0.3924 & 464.24362 & 17.91024 & 67.8097 \\
\hline
\end{tabular}




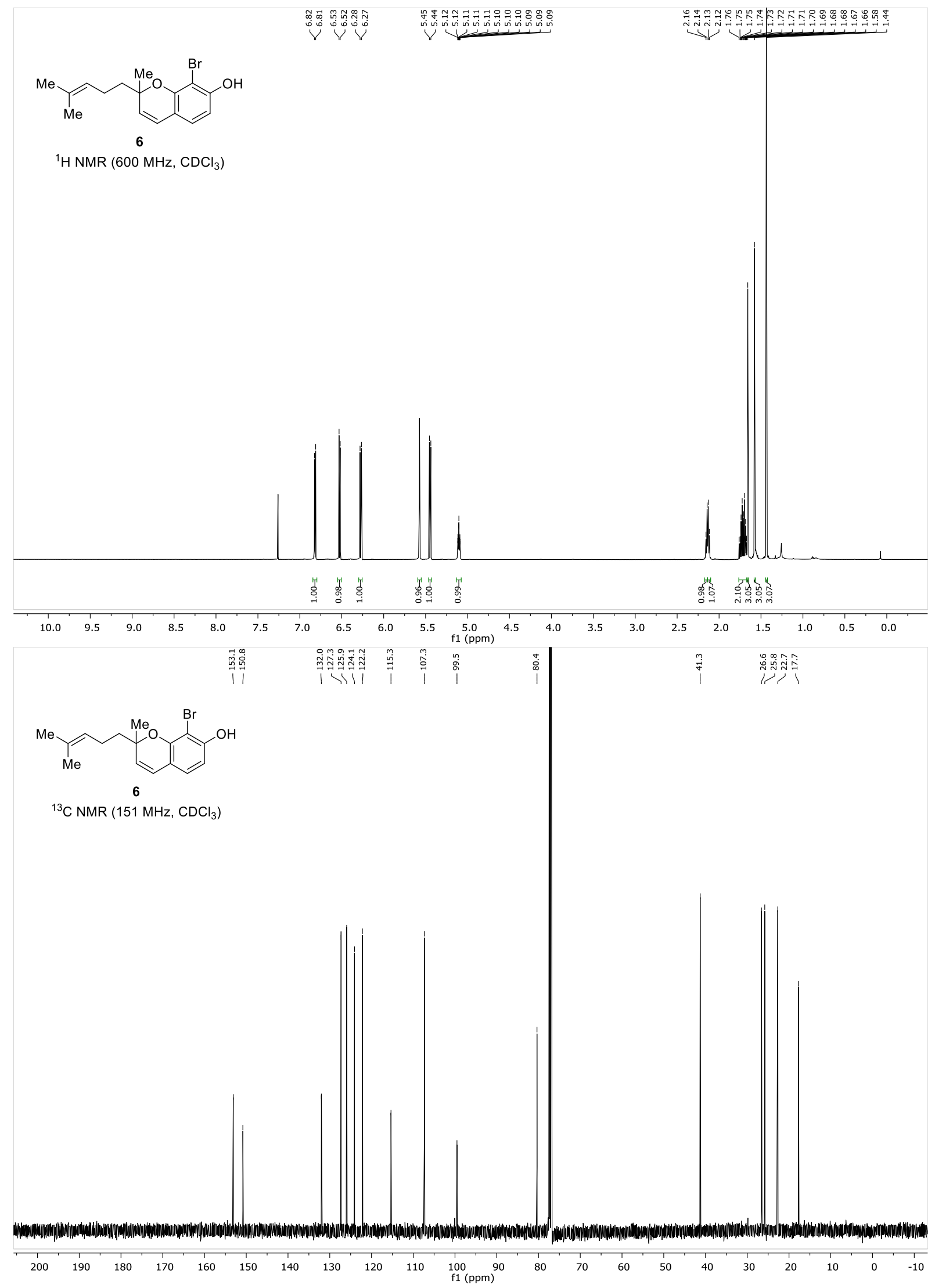




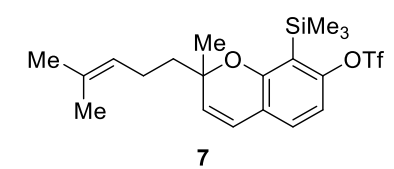

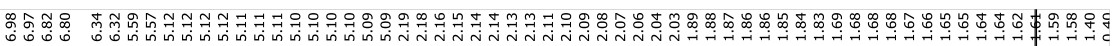

${ }^{1} \mathrm{H}$ NMR $\left(500 \mathrm{MHz}, \mathrm{CDCl}_{3}\right)$

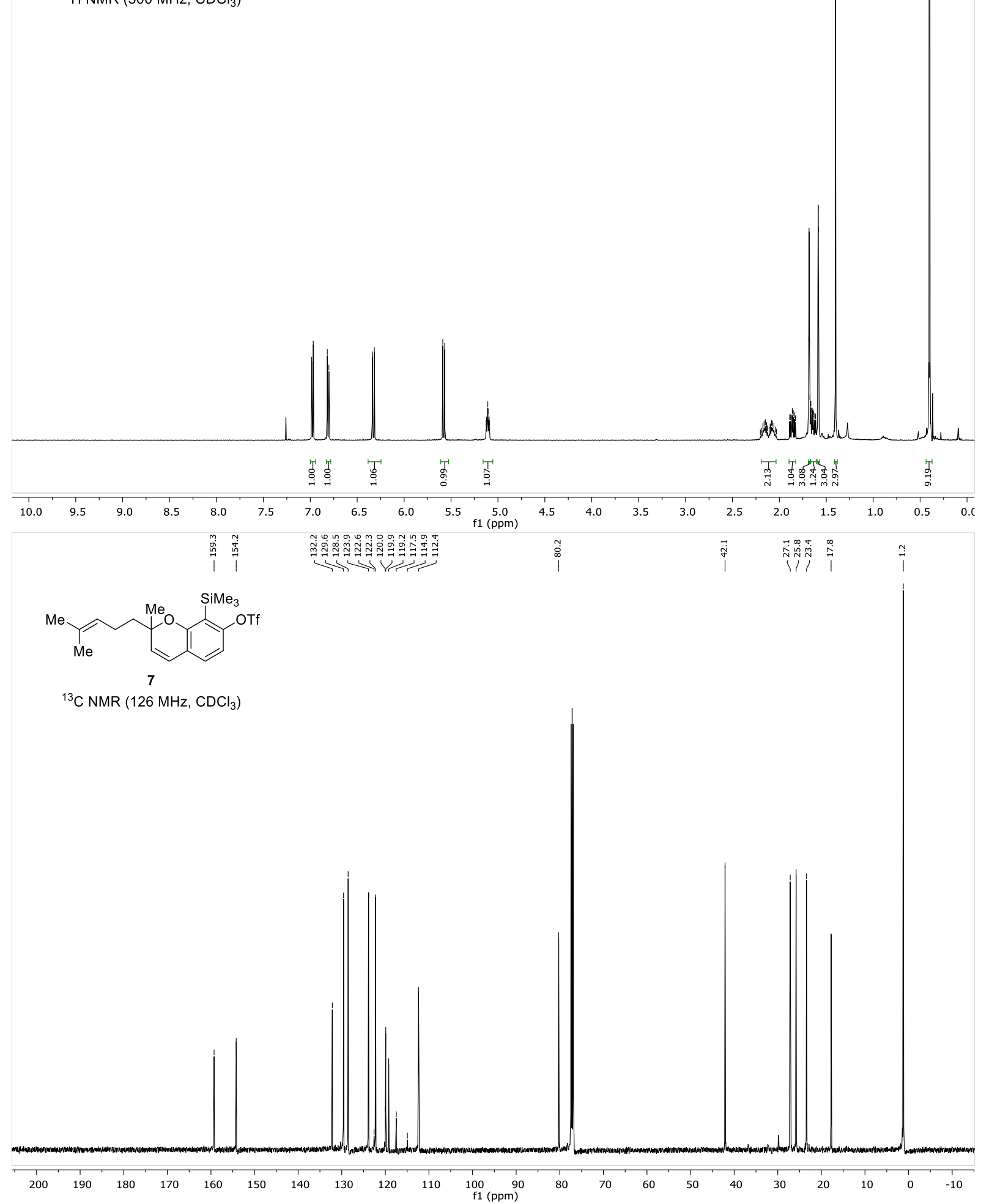




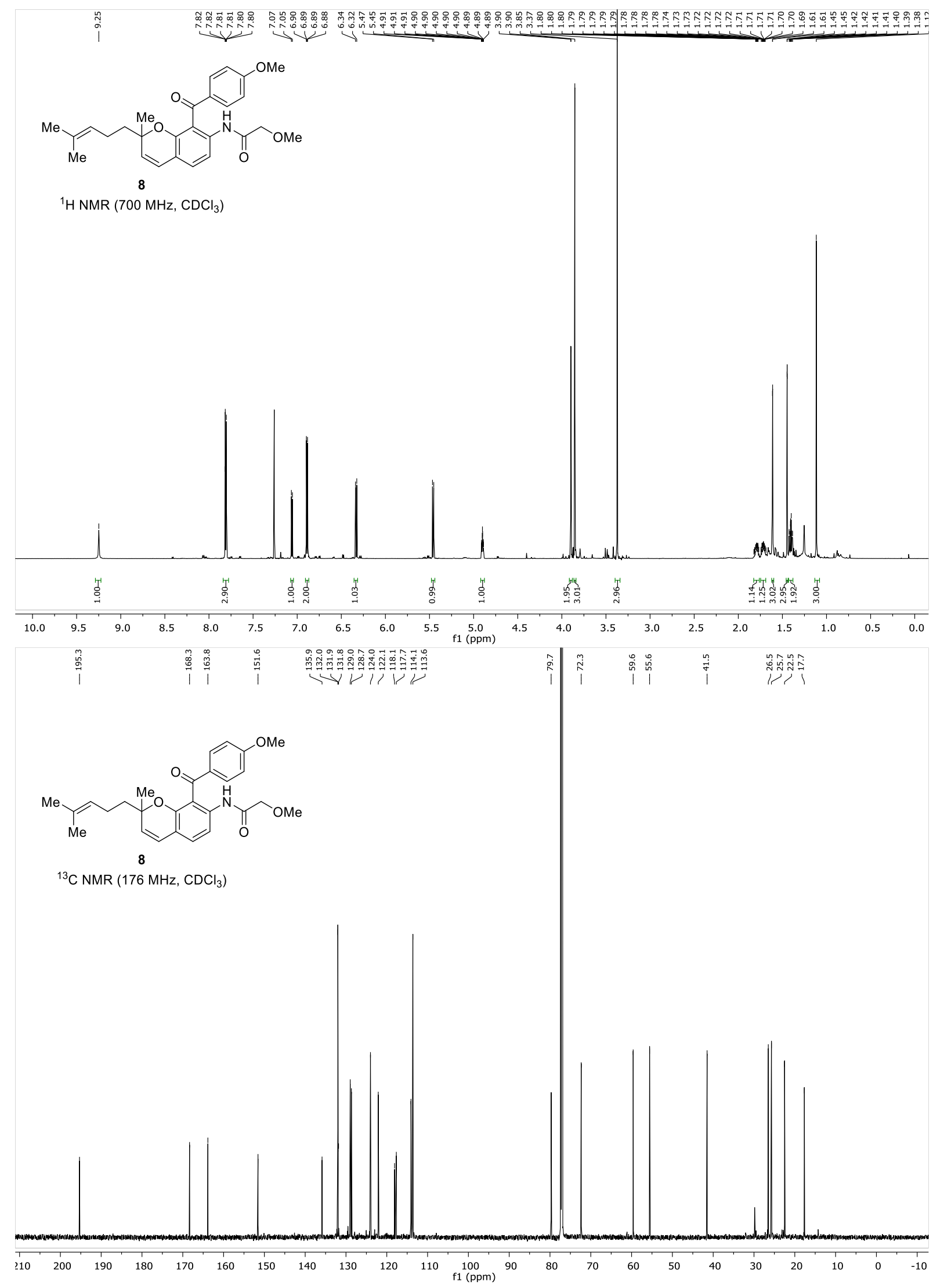




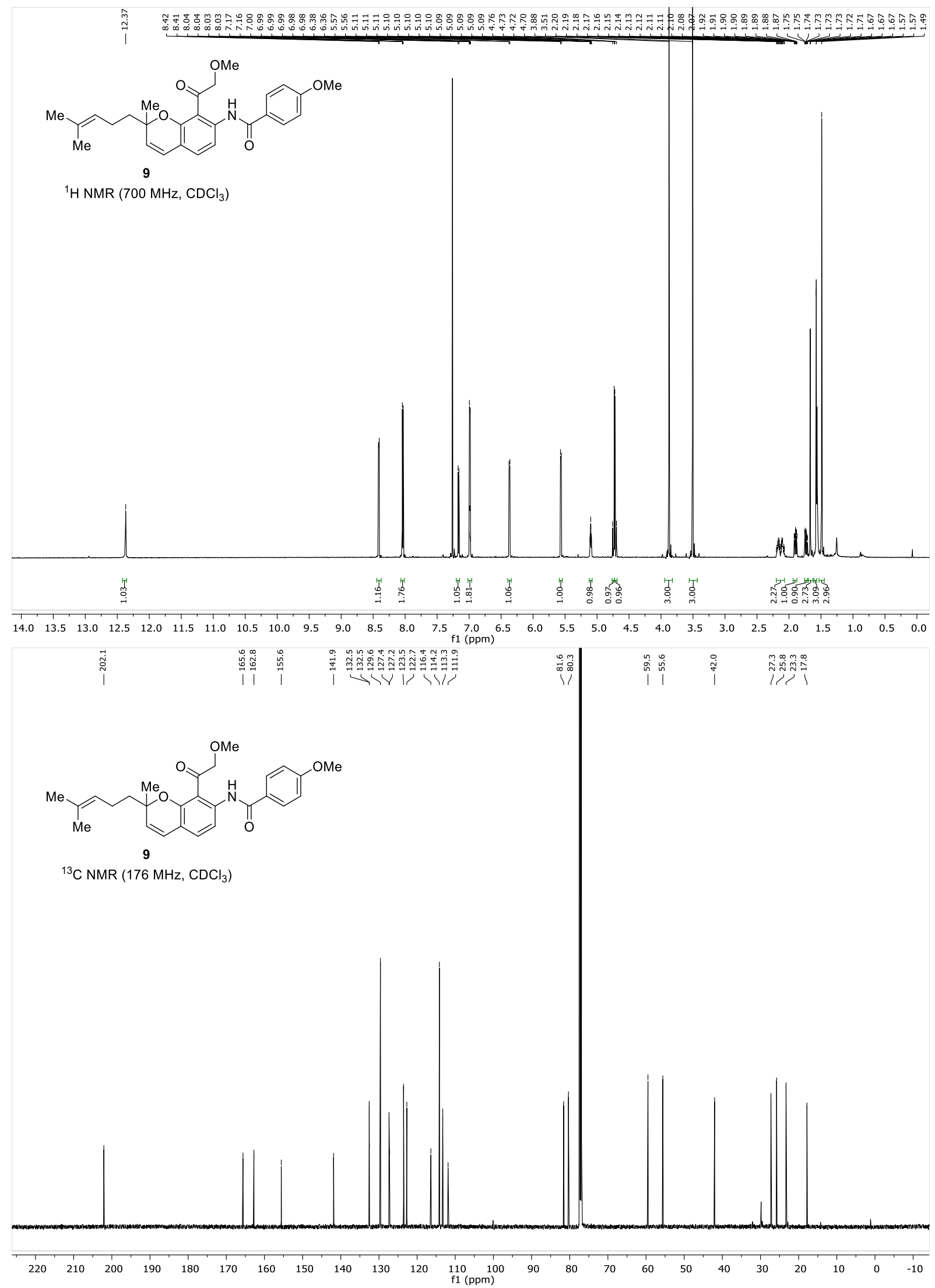




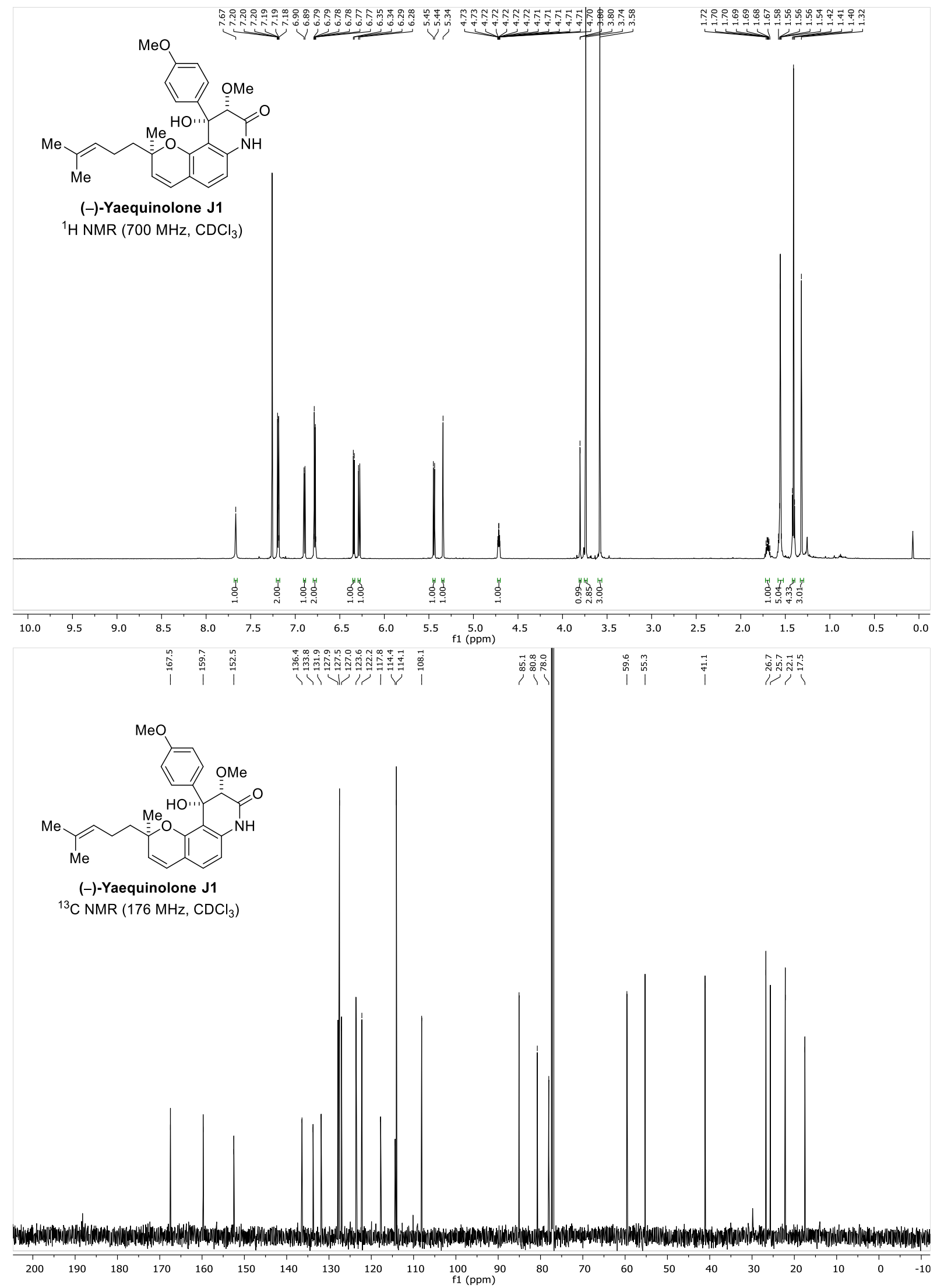




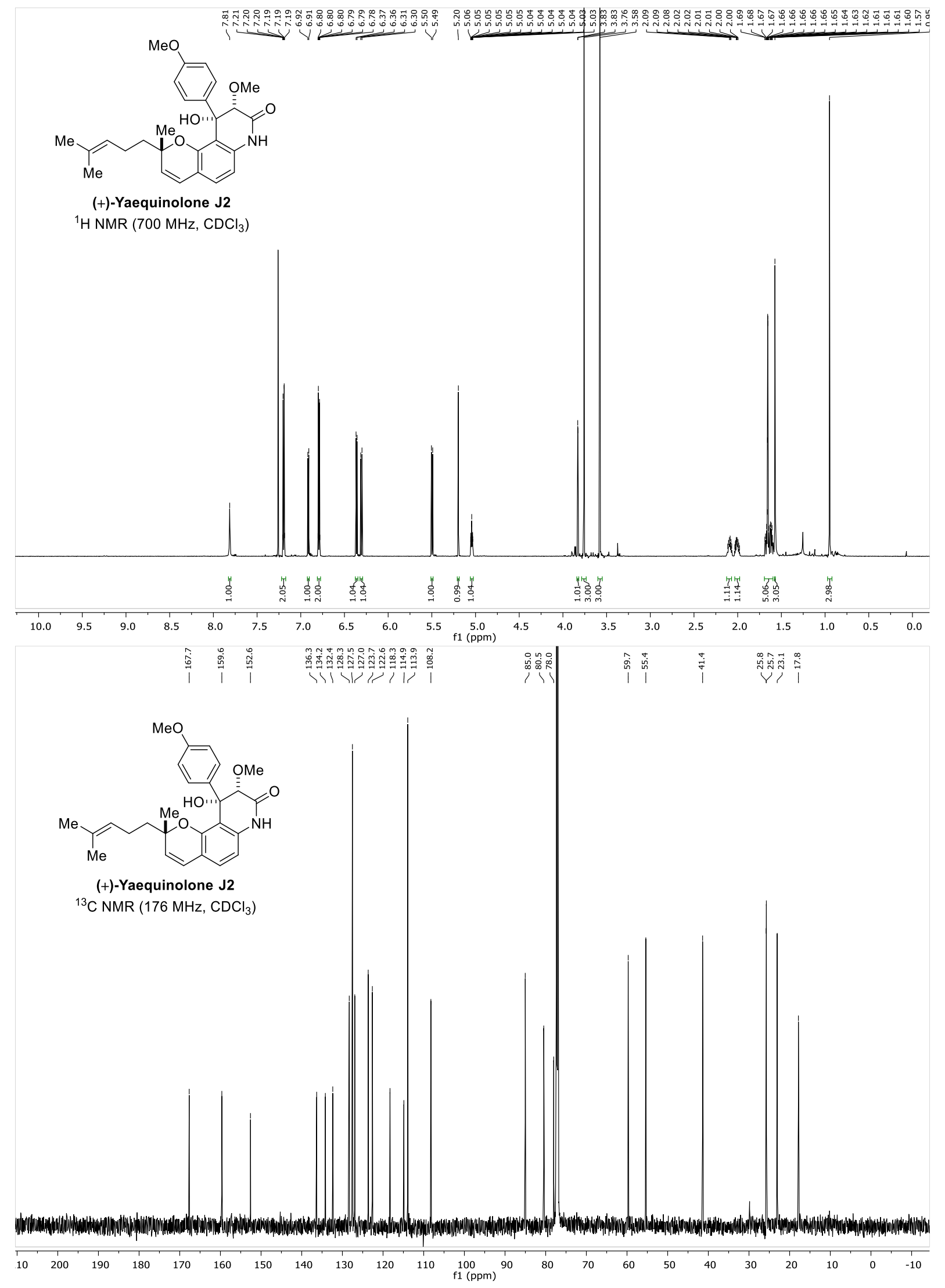

